\title{
Cognitive behavioural therapy improved symptoms in chronic schizophrenia
}

\author{
Tarrier N, Yusupoff L, Kinney C, et al. Randomised controlled trial of intensive cognitive behaviour for patients with chronic \\ schizophrenia. BMJ 1998 Aug 1;317:303-7.
}

\section{Question}

In patients with chronic schizophrenia, is intensive cognitive behaviour therapy (CBT) and routine care superior to supportive counselling and routine care or routine care alone?

\section{Design}

Randomised controlled trial (RCT) with post-treatment assessment at 3 months.

\section{Setting}

Psychiatric outpatient clinics in Manchester, UK.

\section{Patients}

87 patients who were 18-65 years of age and had a diagnosis of schizophrenia, schizoaffective psychosis, or delusional disorder according to the DSM-III-R; hallucinations or delusions for $\geqslant 6$ months; were receiving of stable medication; and had permission from the responsible physician to participate. Exclusion criteria were evidence of organic brain disease; substance abuse was the primary problem; psychological or family intervention; or violence towards the assessors. 72 patients $(83 \%)$ completed the treatment.

\section{Intervention}

Patients were stratified by symptom severity and sex and allocated to intensive CBT plus routine care $(\mathrm{n}=33)$, supportive counselling plus routine care $(n=26)$, or routine care alone $(n=28)$. CBT involved coping strategy enhancement, training in problem solving, and strategies to reduce relapse risk. Supportive counselling involved developing a relationship that fostered rapport and unconditional regard. Routine care was standard psychiatric management with medication and outpatient monitoring.

\section{Main outcome measures}

Number and severity of symptoms, clinical improvement $(\geqslant 50 \%$ improvement in symptoms), relapse, and hospital readmission.

\section{Main results}

CBT led to a decrease from baseline in both number and severity of symptoms (mean decrease in number 1.6, 95\% CI 0.7 to 2.5; mean decrease in severity 7.8, CI 3.8 to 12.0 ). The decreases were greater than those of patients who received supportive counselling. Routine care alone showed slight increases in number and severity of symptoms. Clinical improvement was achieved by more patients who received CBT than patients in the other 2 groups combined $(\mathrm{p}=0.02)$ (table). No patients in either the CBT group or the supportive counselling group had relapses compared with $4(14 \%)$ in the routine care group. The total number of hospital days for routine care group patients was 204 compared with 1 day in each of the CBT and supportive counselling groups.

\section{Conclusions}

Cognitive behaviour therapy plus routine care improved symptoms in chronic schizophrenia more than routine care alone. Supportive counselling improved symptoms to a lesser extent than cognitive behaviour therapy.

Cognitive behaviour therapy $v$ supportive counselling and routine care at 3 months for chronic schizophrenia*

\begin{tabular}{|c|c|c|c|c|}
\hline Outcomes & $C B T$ & $\begin{array}{l}S C \text { and } R C \\
\text { combined }\end{array}$ & $R B I(95 \% C I)$ & $N N T(C I)$ \\
\hline $\begin{array}{l}\text { Clinical improvement } \\
(\geqslant 50 \% \text { improvement in } \\
\text { symptoms })\end{array}$ & $33 \%$ & $13 \%$ & $\begin{array}{l}157 \% \\
\text { (13 to } 487 \text { ) }\end{array}$ & $\begin{array}{l}5 \\
\text { (3 to } 38)\end{array}$ \\
\hline
\end{tabular}

*CBT $=$ cognitive behaviour therapy; $\mathrm{SC}=$ supportive counselling; $\mathrm{RC}=$ routine care. Other abbreviations defined in glossary; RBI, NNT, and CI calculated from data in article.

Source of funding: Wellcome Trust.

For correspondence: Prof N Tarrier, Department of Clinical Psychology, School of Psychiatry and Behavioural Sciences, University of Manchester, Withington Hospital, Manchester M20 8LR, UK. Fax +44 (0)161 2913814 .

\section{Commentary}

The study by Tarrier $e t a l$ is one of the few RCTs of CBT for psychosis. ${ }^{1-3}$

The authors dealt with the methodological requirements of RCTs, taking care to ensure blind assessments and treatment fidelity. They showed that both of these were achieved, which is impressive. They also concentrated on short interventions: 20 hours of CBT delivered over a 10 week period, in keeping with the 6 week intervention from their study publishedin $1993,{ }^{1}$ but in contrast with Kuipers et al who offered 9 months of CBT. ${ }^{2}$ They focused on enhancing coping strategies and problem solving (separate elements of the 1993 study), combining them with relapse prevention. These interventions seem deliberately symptom focused and aimed at symptom reduction rather than at fundamental cognitive change such as reappraisal or schema change. The authors separated particular elements of a CBT approach from such non-specific aspects as interest and positive regard to evaluate effectiveness. They found that CBT had more effect on symptoms, but it is interesting that 20 hours of engagement in talking about difficulties also had positive (non-significant) effects.

Whether such short (and by implication extremely cost effective) intervention maintains improvement is not clear. The methodological rigour of this study augments emerging evidence that psychological interventions are effective in addition to medications for patients with ongoing symptoms of psychosis. Clinically, it adds weight to the view that CBT for psychosis should be offered routinely.

Elizabeth Kuipers, MSc, PhD Institute of Psychiatry London, UK

1 Tarrier N, Beckett R, Harwood S, et al. Br J Psychiatry 1993;162:524-32.

2 Kuipers E, Garety P, Fowler D, et al. Br J Psychiatry 1997;1 171:319-27.

3 Drury V, Birchwood M, Cochrane R, et al. Br J Psychiatry 1996;169:602-7. 